\title{
A FEW REMARKS ON $n$-INFINITE FORCING COMPANIONS
}

\section{Milan Z. Grulović}

\begin{abstract}
We show that the basic properties of Robinson's infinite forcing companions are naturally transmitted to the so called $n$-infinite forcing companions and start with the examination of mutual relations of $n$-infinite forcing companions of Peano arithmetic.
\end{abstract}

\section{Preliminaries}

Throughout the article $L$ is a first order language. In general discussions mostly it is irrelevant whether it is with equality or not; however, in some cases, for instance when it comes to finite models, the supposition of the existence of the equality relation could be of significance - see 2.6.

For a theory $T$ of the language $L, \mu(T)$ will be the slass of all its models (as usual, by a theory we assume a consistent deductively closed set of sentences - thus, $T \vdash \varphi$ means $\varphi \in T)$. By $\Sigma_{n}$-formula we mean any formula equivalent to a formula in prenex normal form whose prenex consists of $n$ blocks of quantifiers, the first one is the block of existential quantifiers ( $\Pi_{n}$-formulas are defined analoguosly). The models (of the language $L$ ) will be denote by $\mathbf{A}, \mathbf{B} \ldots$, while their domains will be $A, B, \ldots$ For a model $\mathbf{A}, \operatorname{Diag}_{n}(\mathbf{A})$ is the set of all $\Sigma_{n^{-}}, \Pi_{n}$-senteneces of the language $L(A)$ (the simple expansion of the language $L$ obtained by adding a new set of constants which is in one to one correspendence with domain $A$ ) which hold in $\mathbf{A}$. In particular, for $n=0, \operatorname{Diag}_{0}(\mathbf{A})$ is not the diagram of $\mathbf{A}$ in the sense in which it is used in model theory, but this difference is of no importance for the text (the same situation we had when we were dealing with the generalization of finite forcing). As usual, we will not distinguish an element $a$ from $A$ and to it the corresponding constant. If $\mathbf{A}$ is a submodel of $\mathbf{B}$ and $(\mathbf{B}, a)_{a \in A} \vDash \operatorname{Diag}_{n}(\mathbf{A})$, we say that $\mathbf{A}$ is an $n$-elementary submodel of $\mathbf{B}$ (i.e., that $\mathbf{B}$ is an $n$-elementary extension of $\mathbf{A}$ ), in notation $\mathbf{A} \prec_{n} \mathbf{B}$. In general, $\mathbf{A}$ is $n$-embedded in $\mathbf{B}$ if for some embedding $f$ of $\mathbf{A}$ into $\mathbf{B}, f(\mathbf{A})$ is an $n$-elementary submodel of $\mathbf{B}$. A $\Sigma_{n+1}$-chain of models is a chain of models $\mathbf{A}_{0}<\mathbf{A}_{1}<\cdots<\mathbf{A}_{\alpha}<\cdots, \alpha<\gamma$, where for each

2000 Mathematics Subject Classification: Primary 03C25; Secondary 03C52, 03C62.

Key words and phrases: Infinite forcing, finite forcing, model companion. 
$\alpha<\beta(<\gamma), \mathbf{A}_{\alpha}$ is an $n$-elementary submodel of $\mathbf{A}_{\beta}$; we use $\mathbf{A}<\mathbf{B}$ to denote that $\mathbf{A}$ is a submodel of $\mathbf{B}$, therefore $<$ is "equal" to $\prec_{0}$.

The definition of $n$-infinite forcing follows the definition of (Robinson's) infinite forcing with the exception of the the case of "negation". So if $\mathcal{K}$ is a class of models of the language $L, \mathbf{A}$ its element and $\varphi$ a sentence of the language $L(A)$, we say that $\mathbf{A} n$-infinitely forces $\neg \varphi$ (with respect to the class $\mathcal{K}$ ), in notation $\mathbf{A} \vDash_{n} \varphi$, iff no $n$-elementary extension of $\mathbf{A} n$-infinitely forces $\varphi$. A model $\mathbf{A}$ from $\mathcal{K}$ is $n$-infinitely generic iff for any sentence $\varphi$ of the language $L(A)$ either $\mathbf{A} \Vdash \vDash_{n} \varphi$ or $\mathbf{A} \Vdash \models_{n} \neg \varphi$.

On condition that $\mathcal{K}$ is $n$-inductive, i.e., closed under unions of $\Sigma_{n+1}$-chains, it holds:

Lemma 1.1. Any model of class $\mathcal{K}$ is an $n$-elementary submodel of some $n$ infinitely generic model.

Lemma 1.2. (a) If $\mathbf{A}$ and $\mathbf{B}$ are $n$-infinitely generic models of the class $\mathcal{K}$ and if $\mathbf{A}$ is an n-elementary submodel of $\mathbf{B}$, then $\mathbf{A}$ is an elementary submodel of $\mathbf{B}$;

(b) every $n$-infinitely generic model of the class $\mathcal{K}$ is an $n$-existentially closed model in $\mathcal{K}$.

THEOREM 1.3. The class $\mathcal{L}_{\mathcal{K}}^{n}$ of all $n$-infinitely generic models of the class $\mathcal{K}$ is the unique subclass $\mathcal{C}$ of the class $\mathcal{K}$ satisfying the following:

(1) $\mathcal{C}$ is $n$-mutually-consistent or, in other words, $n$-model-consistent with $\mathcal{K}$ (which means in fact that any model of $\mathcal{K}$ is an $n$-elementary submodel of some model from $\mathcal{C}$ );

(2) $\mathcal{C}$ is n-model-complete;

(3) $\mathcal{C}$ contains any other subclass of $\mathcal{K}$ satisfying the conditions (1) and (2).

For the readers who are not willing to bother themselves by recalling the basic facts from the theory of infinite forcing the proof of these (and some other) analogies of the corresponding assertions of $n$-infinite forcing are given in $[\mathbf{9}]$.

\section{2. $n$-infinite forcing companion}

Definition 2.1. For a given theory $T$ of the language $L$ let $\mathcal{L}_{T}^{n}$ be the subclass of the $n$-infinitely generic models of the class $\mu\left(T \cap \Pi_{n+1}\right)$. Then the theory $\operatorname{Th}\left(\mathcal{L}_{T}^{n}\right)$ is called the $n$-infinite forcing companion of the theory $T$ and is denoted by $T^{F_{n}}$.

The theory $T$ is $n$-infinite forcing complete iff $T=T^{F_{n}}$.

LEMMA 2.2. (a) If a (first-order) theory $T$ of the language $L$ has the $n$-model companion $-T^{*}$, then $\mathcal{E}_{T}^{n}=\mathcal{L}_{T}^{n}=\mu\left(T^{*}\right)$, where $\mathcal{E}_{T}^{n}$ is the class of $n$-existentially complete models of the theory $T$;

(b) If one of the subclasses $\mathcal{E}_{T}^{n}, \mathcal{L}_{T}^{n}$ of the class $\mu\left(T \cap \Pi_{n+1}\right)$ is a generalized elementary class, then so is the other and in that case these classes have the same theory $\left(\operatorname{Th}\left(\mathcal{E}_{T}^{n}\right)=\operatorname{Th}\left(\mathcal{L}_{T}^{n}\right)\right)$ which is the n-model companion of the theory $T$.

PROOF. We recall that in the case it exists, the $n$-model companion of the theory $T$ is the theory which is $n$-mutually-consistent with $T$ and $n$-model complete. 
(a) It is known that $\mathcal{E}_{T}^{n}=\mu\left(T^{*}\right)$ (see [9], [10]). Thus, $\mathcal{E}_{T}^{n}$ is $n$-model complete and, by $1.2(\mathrm{~b})$ and $1.3, \mathcal{E}_{T}^{n}=\mathcal{L}_{T}^{n}$.

(b) If, for instance, $\mathcal{L}_{T}^{n}$ is a generalized elementary class, then $\operatorname{Th}\left(\mathcal{L}_{T}^{n}\right)$ is $n$ model complete and $n$-mutually-consistent with $T$ (1.3), hence the $n$-model companion of $T$, and, by $(a), \mathcal{L}_{T}^{n}=\mathcal{E}_{T}^{n}$. The similarly case we have when $\mathcal{E}_{T}^{n}$ is a generalized elementary class, only now we use the fact that a theory, whose each model is $\Sigma_{n+1}$-existentially complete is $n$-model complete.

THEOREM 2.3. For given (first-order) theories $(T)$ and their n-infinite forcing companions it holds:

(1) $T^{F_{n}}=\left(T \cap \Pi_{n+1}\right)^{F_{n}}$;

(2) $T \cap \Pi_{n+1}=T^{F_{n}} \cap \Pi_{n+1}$;

(3) $T^{F_{n} F_{n}} \stackrel{\text { def }}{=}\left(T^{F_{n}}\right)^{F_{n}}=T^{F_{n}}$;

(4) $T_{1} \cap \Pi_{n+1}=T_{2} \cap \Pi_{n+1}$ iff $T_{1}^{F_{n}}=T_{2}^{F_{n}}$;

(5) $T^{F_{n}}$ is a complete theory iff $T$ has the $n$-joint embedding property $[8]$;

(6) $T \cap \Pi_{n+2} \subseteq \operatorname{Th}\left(\mathcal{E}_{T}^{n}\right) \subseteq T^{F_{n}}$;

(7) If $T$ has n-model companion $-T^{*}$, then $T^{*}=T^{F_{n}}$.

Proof. (1) A direct consequence of the definition of $n$-infinite forcing companion.

(2) It is clear that $T \cap \Pi_{n+1} \subseteq T^{F_{n}} \cap \Pi_{n+1}$. The other inclusion follows from 1.1; if $\varphi$ is $\Pi_{n+1}$-sentence which is not a consequence of $T$, then for some model $\mathbf{A}$ of $T, \mathbf{A} \vDash \neg \varphi$, and if the an $n$-infinitely generic model $\mathbf{B}$ is an $n$-elementary extension of $\mathbf{A}$, it follows $\mathbf{B} \vDash \neg \varphi$, whence $\varphi \notin T^{F_{n}}$.

(3) By the previous items we have:

$$
T^{F_{n} F_{n}}=\left(T^{F_{n}}\right)^{F_{n}}=\left(T^{F_{n}} \cap \Pi_{n+1}\right)^{F_{n}}=\left(T \cap \Pi_{n+1}\right)^{F_{n}}=T^{F_{n}} .
$$

(4) An immediate consequence of (1) and (2).

(5) Suppose that $T$ has the $n$-joint embedding property but that $T^{F_{n}}$ is not complete. Then, for some sentence $\varphi$ of the language $L$ and some $n$-infinitely generic models $\mathbf{A}$ and $\mathbf{B}, \mathbf{A} \vDash \varphi$ and $\mathbf{B} \vDash \neg \varphi$. Let $\mathbf{A}_{1}$ and $\mathbf{B}_{1}$ be models of $T$, which are, respectively, $n$-elementary extensions of $\mathbf{A}$ and $\mathbf{B}$ (such models exist since $\left.\mathbf{A}, \mathbf{B} \in \mu\left(T \cap \Pi_{n+1}\right)\right)$. Let futher $\mathbf{C}$ be a model of $T$ into which the models $\mathbf{A}_{1}$ and $\mathbf{B}_{1}$ are $n$-embedded and let $\mathbf{D}$ be an $n$-infinitely generic model which is an $n$-extension of $\mathbf{C}$. Then $\mathbf{A}$ and $\mathbf{B}$ are elementary embedded into $\mathbf{D}$, hence $\mathbf{D}$ satisfies both $\varphi$ and $\neg \varphi$, a contradiction. The other implication is also obvious; we use the facts that any complete theory has the $n$-joint embedding property and that $\mathcal{L}_{T}^{n}$ is $n$-model consistent with $\mu\left(T \cap \Pi_{n+1}\right)$.

(6) The relation $T \cap \Pi_{n+2} \subseteq \operatorname{Th}\left(\mathcal{E}_{T}^{n}\right)$ is already known and the relation $\operatorname{Th}\left(\mathcal{E}_{T}^{n}\right) \subseteq T^{F_{n}}$ follows directly from $1.2(b)$.

(7) By the previous lemma, $\mathcal{L}_{T}^{n}=\mu\left(T^{*}\right)$, whence $T^{F_{n}}=\operatorname{Th}\left(\mu\left(T^{*}\right)\right)=T^{*}$.

Corollary 2.4. (a) If $T$ is a complete, $n$-inductive theory, then $T=T^{F_{k}}$ for each $k \geqslant n$;

(b) If for some $n, T^{F_{n}}=T^{F_{k}}$ for each $k>n$, then $T=T^{F_{n}}$; 
(c) If for some $n>0, T^{F_{n}}$ is a complete theory, then, for each $k<n, T^{F_{k}}$ is also complete.

LEMMA 2.5. Let $\mathbf{A}$ be a model of $T \cap \Pi_{n+1}$. If the theory $\left(T \cap \Pi_{n+2}\right) \cup \operatorname{Diag}_{n}(\mathbf{A})$ is complete (i.e., if $\mathbf{A} n$-completes $T \cap \Pi_{n+2}$ ), then $\mathbf{A}$ is an $n$-infinitely generic model.

Proof. Trivial. There is some $n$-infinitely generic model $\mathbf{B}$ which is also an $n$-elementary extension of $\mathbf{A}$. By condition of the lemma, $\mathbf{A}$ is an elementary submodel of $\mathbf{B}$, thus itself an $n$-infinitely generic model.

Corollary 2.6. Let $T$ be a theory defined in the language $(L)$ with equality and with finite models. Then any its finite model is $n$-infinitely generic for any $n \geqslant 1$.

Proof. Let $\mathbf{A}$ be a finite model of $T$. By a $\Pi_{1}$-sentence from $\operatorname{Diag}_{1}(\mathbf{A})$ we can say that $\mathbf{A}$ has exactly $|A|$ elements, so the only $n$-elementary extension of $\mathbf{A}$ (for $n \geqslant 1$ ) is $\mathbf{A}$ itself.

COROLlary 2.7. (a) If $T$ is an $n$-inductive theory and for each sentence $\varphi$ consistent with $T$ there exists a model of $T \cup\{\varphi\}$ which n-completes $T$, then $T$ is $n$-infinite forcing complete.

(b) Any $n$-model complete theory is $n$-infinite forcing complete and, in fact, it is its own n-model completion.

Proof. (a) By 2.3(6), $T \subseteq T^{F_{n}}$. Suppose $\varphi \notin T$. Then, by the condition of the corollary, there exists a model $\mathbf{A}$ of $T \cup\{\neg \varphi\}$ which $n$-completes $T$ and thus it is $n$-infinitely generic. It follows that $\varphi$ is not in $T^{F_{n}}$.

Part (b) is a direct consequence of $(a)$ and the uniqueness of $n$-companion operator.

LEMMA 2.8. Let $\mathbf{A}$ be a model of $T \cap \Pi_{n+1}$ and $\bar{a}$ an enumeration of its elements. Further, let $\|_{n}$ and $\|_{n}^{A}$ be the $n$-infinite forcing relations corresponding, respectively, to the theories $T$ and $T \cup \operatorname{Diag}_{n}(\mathbf{A})$. Then:

(a) If $\mathbf{B}$ is an $n$-elementary extension of $\mathbf{A}$ in $\mu\left(T \cap \Pi_{n+1}\right)$ and $\varphi$ a sentence defined in $\mathbf{B}$, then

$$
\mathbf{B} \models_{n} \varphi \quad \text { iff }(\mathbf{B}, \bar{a}) \models_{n}^{A} \varphi ;
$$

in particular, $\mathbf{B} \in \mathcal{L}_{T}^{n}$ iff $(\mathbf{B}, \bar{a}) \in \mathcal{L}_{T \cup \operatorname{Diag}_{n}(\mathbf{A})}^{n}$;

(b) $T^{F_{n}} \cup \operatorname{Diag}_{n}(\mathbf{A}) \subseteq\left(T \cup \operatorname{Diag}_{n}(\mathbf{A})\right)^{F_{n}}$.

ProOF. (a) By induction on the complexity of the formula $\varphi$. Obviously, only the case $\varphi \equiv \neg \psi$ is of some interest. Suppose $\mathbf{B} \|_{n} \varphi$, but not $(\mathbf{B}, \bar{a}) \|_{n}^{A} \varphi$. Then, for some $n$-elementary extension $(\mathbf{C}, \bar{a})$ of $(\mathbf{B}, \bar{a})$ in $\mu\left(\left(T \cup \operatorname{Diag}_{n}(\mathbf{A})\right) \cap \Pi_{n+1}\right)$, $(\mathbf{C}, \bar{a}) \models_{n}^{A} \psi$, whence, by inductive hypothesis, $\mathbf{C} \vDash_{n} \psi$. But, because of $\mathbf{B} \prec_{n} \mathbf{C}$, C $n$-infinitely forces $\neg \psi$ as well, a contradiction. The inverse implication is clear too.

(b) Suppose that a sentence $\varphi$ of the language $L(A)$ is not in $\left(T \cup \operatorname{Diag}_{n}(\mathbf{A})\right)^{F_{n}}$. Then, for some $(\mathbf{B}, \bar{a}) \in \mathcal{L}_{T \cup \operatorname{Diag}_{n}(\mathbf{A})}^{n},(\mathbf{B}, \bar{a}) \vDash \neg \varphi$, i.e. $(\mathbf{B}, \bar{a}) \vDash_{n}^{A} \neg \varphi$. Hence, 
$\mathbf{B} \vDash_{n} \neg \varphi$, i.e. $\mathbf{B} \vDash \neg \varphi$. Since $\mathbf{B}$ is a model of $T^{F_{n}} \cup \operatorname{Diag}_{n}(\mathbf{A})$, it follows that $\varphi \notin T^{F_{n}} \cup \operatorname{Diag}_{n}(\mathbf{A})$.

Corollary 2.9. If $\mathbf{A}$ is an n-infinitely generic model, then $\left(T \cup \operatorname{Diag}_{n}(\mathbf{A})\right)^{F_{n}}=$ $\operatorname{Th}((\mathbf{A}, \bar{a}))$ - the complete diagram of $\mathbf{A}(\bar{a}$ is still an enumeration of the elements of $\mathbf{A})$.

Proof. Directly, by 1.2(a) and the previous lemma (again the item $(a)$ ).

The next two propositions have the equivalents in the theory of $n$-finite forcing (see [7]) and the proofs of the equivalent assertions are based on the same facts for they are direct consequences of Theorem 2.3 which has the analogy in $n$-finite forcing.

LEMMA 2.10. Let $T_{D L O M}$ be the theory of dense linear order with minimal and maximal elements defined in the language with equality and just one binary relation. Then $T_{D L O M}=T_{D L O M}^{F_{n}}$ for any $n \geqslant 1$ and $T_{D L O M}^{F} \stackrel{\text { def }}{=} T_{D L O M}^{F_{0}}$ is the theory of dense linear order without minimal and maximal elements.

Proof. $T_{D L O M}$ is a complete, $\Pi_{3}$-axiomatizable theory, whence $T_{D L O M}=$ $T_{D L O M}^{F_{n}}$ for each $n \geqslant 1$. On the other side, the existentially closed models of this theory are exactly dense linear orderings without endpoints, which implies the second part of the proposition $(2.2(\mathrm{~b}))$.

Theorem 2.11. Let $T_{P A}$ be Peano arithmetic (defined in the language $L=$ $\{+, \cdot,=, 0,1\})$. Then:

(a) For each (natural number) $n$ there exists (a natural number) $k>n$ such that $T_{P A}^{F_{n}} \neq T_{P A}^{F_{k}}$;

(b) None of the theories $T_{P A}^{F_{n}}, n \geqslant 0$, is complete;

(c) The standard model of Peano arithmetic $(\mathbf{N})$ is not infinitely generic model;

(d) None of the theories $T_{P A}^{F_{n}}, n \geqslant 0$ is model complete; in paricular $T_{P A}^{F}$ is not a model companion of $T_{P A}$.

Proof. (a) Let us suppose that for some $n$ it holds: for any $k>n, T_{P A}^{F_{n}}=$ $T_{P A}^{F_{k}}$. Then by $2.4(b), T_{P A}=T_{P A}^{F_{n}}$. By $1.2(b)$, there exists a nonstandard model of Peano arithmetic which is $n$-existentially complete, contradictory to Theorem 5 from $[4]$.

(b) It is known that Peano arithmetic does not have the joint embedding property (see $[\mathbf{1 1}]$, or, for more details $[\mathbf{1 4}],[\mathbf{1 5}],[\mathbf{1 6}]$ ).

(c) Due to (one) Gödel's result, for any subtheory $T$ of $\mathrm{Th}(\mathbf{N})$ with an effectively recursively enumerable set of axioms there exists an universal sentence which is satisfied in $\mathbf{N}$ but does not belong to $T$. Let us take for $T$ the $\Pi_{2}$-segment of Peano arithmetic and let $\varphi$ be an universal sentence from $\operatorname{Th}(\mathbf{N})$ which is not in $T_{P A} \cap \Pi_{2}$. If we supposed that $\mathbf{N}$ is an infinitely generic model, then, by $1.2(a)$, it would follow that $\varphi \in T_{P A}^{F}$, whence (by 2.3(2)) $\varphi \in T_{P A}$, a contradiction.

(d) In [5, Theorem 3.1] it is shown: no theory extending $\Pi_{2}$-segment of Peano arithmetic is model complete. 
Let us still note the following. It is known that $\mathbf{N}$ is the only finitely generic model of the (finitely forcing complete) theory $\operatorname{Th}(\mathbf{N})$ defined in the language $L=\{+, \cdot,=, 0,1\}[\mathbf{1}$, Theorem 5.7]. On the other side, $\mathbf{N}$ is not infinitely generic model for the same theory. Otherwise, by $1.2(\mathrm{a}), \operatorname{Th}(\mathbf{N})$ would be infinitely forcing complete, but no existentially closed model of $\operatorname{Th}(\mathbf{N})$, except $\mathbf{N}$, is model of Peano arithmetic (see Theorems 2.20 and 2.21 in [17]). This example shows that in general the condition in Lemma 2.5 cannot be weakened: $T \cup \operatorname{Diag}_{n}(\mathbf{A})$ is complete does not necessarily imply that $\mathbf{A}$ is $n$-infinitely generic (but it implies that $\mathbf{A}$ is $n$-finitely generic model - see the proof of Theorem 4.9 in [1] and Theorem 0.1 in $[7])$.

By 2.5 and 2.3(6), any finite model in the theory with equality and with finite models, which includes a lot of interesting theories - groups, rings, fields and so on, is both 1-finitely and 1-infinitely generic model and, surely, in the cases of mentioned theories it is neither finitely nor infinitely generic model. Thus for these theories the intersection of the classes of 1-finitely and 1-infinitely generic models is nonempty. However, the classes of finitely and infinitely generic models can be disjoint, for instance in the case of groups [13, Theorem 11], commutative rings with identity [3, Theorem 61] etc. It follows that for these theories infinite and 1-infinite forcing companions do not coincide (the first one contains the sentences which provide the existence of infinitely many elements). Of course, in the case the theory has the joint embedding property, but not the 1-joint embedding property we obtain immediately that the infinite forcing companion is complete, while the 1-infinite forcing companion is not complete.

\section{References}

[1] J. Barwise, A. Robinson, Completing Theories by Forcing, Annals of Mathematical Logic Vol. 2, No. 2 (1970), 119-142.

[2] C. C. Chang, H. J. Keisler, Model Theory, North-Holland, Amsterdam - London, 1973.

[3] G. Cherlin, Model Theoretic Algebra - Selected Topics, Lecture Notes in Mathematics 521, Springer-Verlag, Berlin-Heidelberg-New York, 1976.

[4] H. Gaifman, A Note on Models and Submodels of Arithmetic, Lecture Notes in Mathematics 255, Springer-Verlag, 1972, 128-144.

[5] D. C. Goldrei, A. Macintyre, H. Simmons, The Forcing Companions of Number Theories, Israel J. Math., Vol. 14, 1973, 317-337.

[6] M.Z. Grulović, On n-Finite Forcing, Review of Research, Faculty of Science, University of Novi Sad, Mathematics Series, Vol. 13 (1983), 405-421.

[7] M.Z. Grulović, On n-Finite Forcing Companions, Review of Research, Faculty of Science, University of Novi Sad, Mathematics Series, 14, 2, 1984., 211-222.

[8] M.Z. Grulović, A Word on the Joint Embedding Property, Matematički vesnik 27 (1995), $39-47$.

[9] M. Z. Grulović, A Word on n-Infinite Forcing, (in print)

[10] J. Hirschfeld, W. H. Wheeler, Forcing, Arithmetic, Division Rings, Lecture Notes in Mathematics 454, Springer-Verlag, Berlin-Heidelberg-New York, 1975.

[11] A. Ignjatović, M. Z. Grulović, A Comment on the Joint Embedding Property, Periodica Mathematica Hungarica Vol. 33 (1), (1996), 45-50.

[12] H. J. Keisler, Forcing and Omitting Types Theorem, Studies in Mathematics (ed. A. Morley), Vol. 8, 96-133.

[13] A. Macintyre, On Algebraically Closed Groups, Annals of Mathematics, Vol. 96 (1972), 53-97. 
[14] A. Macintyre, H. Simmons, Algebraic Properties of Number Theories, Israel J. Math., Vol. 22, No. 1, 1975, 7-27.

[15] M. Otero, A Note on the Joint Embedding Property in Fragments of Arithmetic, Bull. London Math. Soc. 24 (1992), 417-423.

[16] M. Otero, The joint embedding property in normal open induction, Annals of Pure and Apllied Logic 60 (1993), 275-290.

[17] A. Robinson, Nonstandard Arithmetic and Generic Arithmetic, Proc. of the Fourth Internat. Congress for Logic, Methodology and Philosophy of Science, Buchares 1971, North-Holland, 1973.

Prirodno-matematički fakultet

21000 Novi Sad

Serbia

gruja@im.ns.ac.yu 